\title{
Correlation of SASHI expression and ultrasonographic features in breast cancer
}

This article was published in the following Dove Press journal:

OncoTargets and Therapy

10 January 2017

Number of times this article has been viewed

\author{
Xuchu Gong' \\ Jinna $\mathrm{Wu}^{\prime}$ \\ Jian $\mathrm{Wu}^{2}$ \\ Jun $\mathrm{Liu}^{2}$ \\ Hailin $\mathrm{Gu}^{\prime}$ \\ Hao Shen ${ }^{3}$ \\ 'Department of Surgery, ${ }^{2}$ Department \\ of Endocrinology, ${ }^{3}$ Department of \\ Doppler Ultrasonic, Traditional \\ Chinese Medicine Hospital of \\ Nantong City, Nantong, Jiangsu, \\ People's Republic of China
}

Objective: SASH1 is a member of the SH3/SAM adapter molecules family and has been identified as a new tumor suppressor and critical protein in signal transduction. An ectopic expression of SASH1 is associated with decreased cell viability of breast cancer. The aim of this study was to explore the association between SASH1 expression and the ultrasonographic features in breast cancer.

Patients and methods: A total of 186 patients diagnosed with breast cancer were included in this study. The patients received preoperative ultrasound examination, and the expression of SASH1 was determined using immunohistochemistry methods. Spearman's rank correlation analysis was used to analyze the correlation between SASH1-positive expression and the ultrasonographic features.

Results: The positive expression of SASH1 was observed in 63 (33.9\%) patients. The positive expression rate of SASH1 was significantly decreased in patients with breast cancer $(63 / 186$, $33.9 \%$ ) compared with controls $(P<0.001)$. The positive expression rate of SASH1 was significantly decreased in patients with edge burr sign $(P=0.025)$, lymph node metastasis $(P=0.007)$, and a blood flow grade of III $(P=0.013)$ compared with patients without those adverse ultrasonographic features. The expression of SASH1 was negatively correlated with edge burr sign $(P=0.025)$, lymph node metastasis $(P=0.007)$, and blood flow grade $(P=0.003)$ of the patients with breast cancer.

Conclusion: The expression of SASH1 was inversely correlated with some critical ultrasonographic features, including edge burr sign, lymph node metastasis, and blood flow grade in breast cancer, and decreased SASH1 expression appears to be associated with adverse clinical and imaging features in breast cancer.

Keywords: SASH1, breast cancer, ultrasound

\section{Introduction}

Breast cancer is one of the most common malignancies affecting women worldwide, and its initiation and development are affected by many factors. ${ }^{1,2}$ Most patients have already been diagnosed in tumor progressing stage when visiting doctors, few of whom are noted in early tumor stage and receive treatment, which would have adverse effects on the patients' prognosis. The current clinical therapy for treating breast cancer is mainly based on the estrogen receptor (ER) levels and pathological staging. ${ }^{3,4}$ Analyzing the correlation between breast ultrasound imaging and specific protein expression in cancerous tissues could contribute to providing a valuable reference for patients' postoperative treatment and prognosis.

SASH1 is a member of SH3/SAM adapter molecules family and has been identified as a new tumor suppressor and critical protein in signal transduction. Ren et $\mathrm{al}^{5}$ reported that downregulated expression of SASH1 inhibited growth, proliferation,
Correspondence: Hao Shen

Department of Doppler Ultrasonic,

Traditional Chinese Medicine Hospital

of Nantong City, $4 \mathrm{I}$ Jianshe Road,

Chongchuan, Nantong 22600I,

Jiangsu, People's Republic of China

Tel +86 I39 I229 2396

Fax +8651385126056

Email shenhaotcmnt@163.com
OncoTargets and Therapy 2017:10 27I-276

(c) $(1) \circledast \odot 2017$ Gong et al. This work is published and licensed by Dove Medical Press Limited. The full terms of this license are avalable at https//www.dovepress.com/terms.php

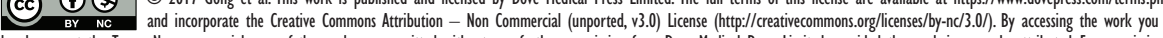
hereby accept the Terms. Non-commercial uses of the work are permitted without any further permission from Dove Medical Press Limited, provided the work is properly attributed. For permision for commercial use of this work, please see paragraphs 4.2 and 5 of our Terms (htpps://www.dovepress. com/terms.php). 
and migration and enhanced apoptosis in ovarian carcinoma. Recently, Burgess et al reported that SASH1 had a subtypedependent effect on the progression of breast cancer and was also associated with its prognosis. Restoring SASH1 protein expression in breast cancer cells through chloropyramine treatment seems to increase apoptosis in breast cancer cells, suggestive of its potential role as a therapeutic target. ${ }^{6}$ In addition, decreased expression of SASH1 is found in $74 \%$ of breast tumors compared with normal breast epithelial tissues. ${ }^{7}$

In this study, SASH1 expression in breast cancer was determined, and its correlation with the ultrasonographic features was analyzed to provide clinical information for treatment and prognosis.

\section{Patients and methods Patients}

A total of 186 patients diagnosed with breast cancer were included in the Traditional Chinese Medicine Hospital of Nantong City from March 2010 to May 2012. The patients received preoperative ultrasound examination and were then confirmed by the pathological examination. Inclusion criteria were 1) initial and untreated tumors, 2) no tumors in other body parts, and 3) diagnosed with single tumor. Exclusion criteria were 1) recurrent tumors and 2) accompanied by tumors in other body parts. The age of the patients was $49.32 \pm 5.78$ years (range 29-75 years). The pathological types included infiltrating ductal carcinoma (153 cases, 82.26\%), invasive lobular carcinoma (18 cases, $9.68 \%$ ), invasive cribriform carcinoma ( 9 cases, $4.84 \%$ ), and mucinous carcinoma (6 cases, 3.23\%). Another 50 patients with ductal epithelial hyperplasia disease, aged $48.57 \pm 6.52$ years (range $28-72$ years), served as controls. All of the subjects provided written informed consent. The study protocols followed the Declaration of Helsinki and were approved by the ethics committee of the Traditional Chinese Medicine Hospital of Nantong City.

\section{Ultrasonography}

Patients took supine position to completely expose bilateral breasts and armpits, and then the successional radial scanning centering on the nipples was conducted; moreover, vertical and horizontal continuous scanning was also conducted based on each quadrant of the breast. In the first place, observations such as the location, size, margin, shape, aspect ratio, internal echo, posterior echo, and calcification status were acquired on two-dimensional ultrasound, then the blood flow in tumor tissues was detected by color Doppler imaging, and the blood flow was classified according to Alder semiquantitative classification standard. The breast lesion was described and classified based on the Breast Imaging and Reporting Data System (BI-RADS) formulated by the American College of Radiology. ${ }^{8}$

\section{Immunohistochemistry}

Briefly, the formalin-fixed and paraffin-embedded tissue sections of $5 \mu \mathrm{m}$ thickness were deparaffinized and then rehydrated with an ethanol gradient solution. Antigen retrieval was performed in a $0.01 \mathrm{~mol} / \mathrm{L}$ sodium citrate buffer at $\mathrm{pH} 6.0$ with a high pressure for $5 \mathrm{~min}$. The tissue sections were fully immersed in $3 \% \mathrm{H}_{2} \mathrm{O}_{2}$ solution for $10 \mathrm{~min}$ to disable endogenous peroxidase activities. Next, the tissue sections were blocked with $10 \%$ goat serum for $30 \mathrm{~min}$ at $37^{\circ} \mathrm{C}$. After that, these sections were incubated with rabbit anti-SASH1 polyclonal antibody (Abcam, Cambridge, MA, USA) for overnight at $4^{\circ} \mathrm{C}$ and further incubated with a secondary antibody against rabbit immunoglobulins (EnVision, DAKO, Denmark) at $37^{\circ} \mathrm{C}$ for another $30 \mathrm{~min}$. Finally, the sections were stained with diaminobenzidine for $5 \mathrm{~min}$. The cytoplasm and cell membrane stained in yellow brown pattern were defined as immune positive. Five representative areas were randomly examined under a microscope at a final magnification of $400 \times$. The number of positive cells $<10 \%$ was defined as negative, and the positive cells $\geq 10 \%$ was defined as positive. The immunohistochemical staining in this study was scored by two pathologists blinded to the clinicopathological data, and a controversy was resolved by consensus opinion with another senior pathologist.

\section{Statistical analysis}

SPSS 19.0 statistical software (SPSS Inc., Chicago, IL, USA) was used for statistical analyses. Categorical variables are presented as rate and constituent ratio, which was analyzed by $\chi^{2}$ test. Spearman's rank correlation analysis was used to analyze the correlation between SASH1 positive expression and the ultrasonographic features. $P<0.05$ was considered statistically significant.

\section{Results \\ Basic characteristics of the patients with breast cancer}

In 186 patients with breast cancer, $75(40.3 \%)$ had a tumor size of $\geq 2 \mathrm{~cm}$ and $111(59.7 \%)$ had a tumor size of $<2 \mathrm{~cm}$. Edge burr sign was found in $118(63.4 \%)$ patients (Figure 1A). A total of 33 (17.7\%) patients had regular shape of the tumors, and the other $153(82.3 \%)$ patients had irregular shape of the tumors. Internal calcification was observed in 90 (48.4\%) 

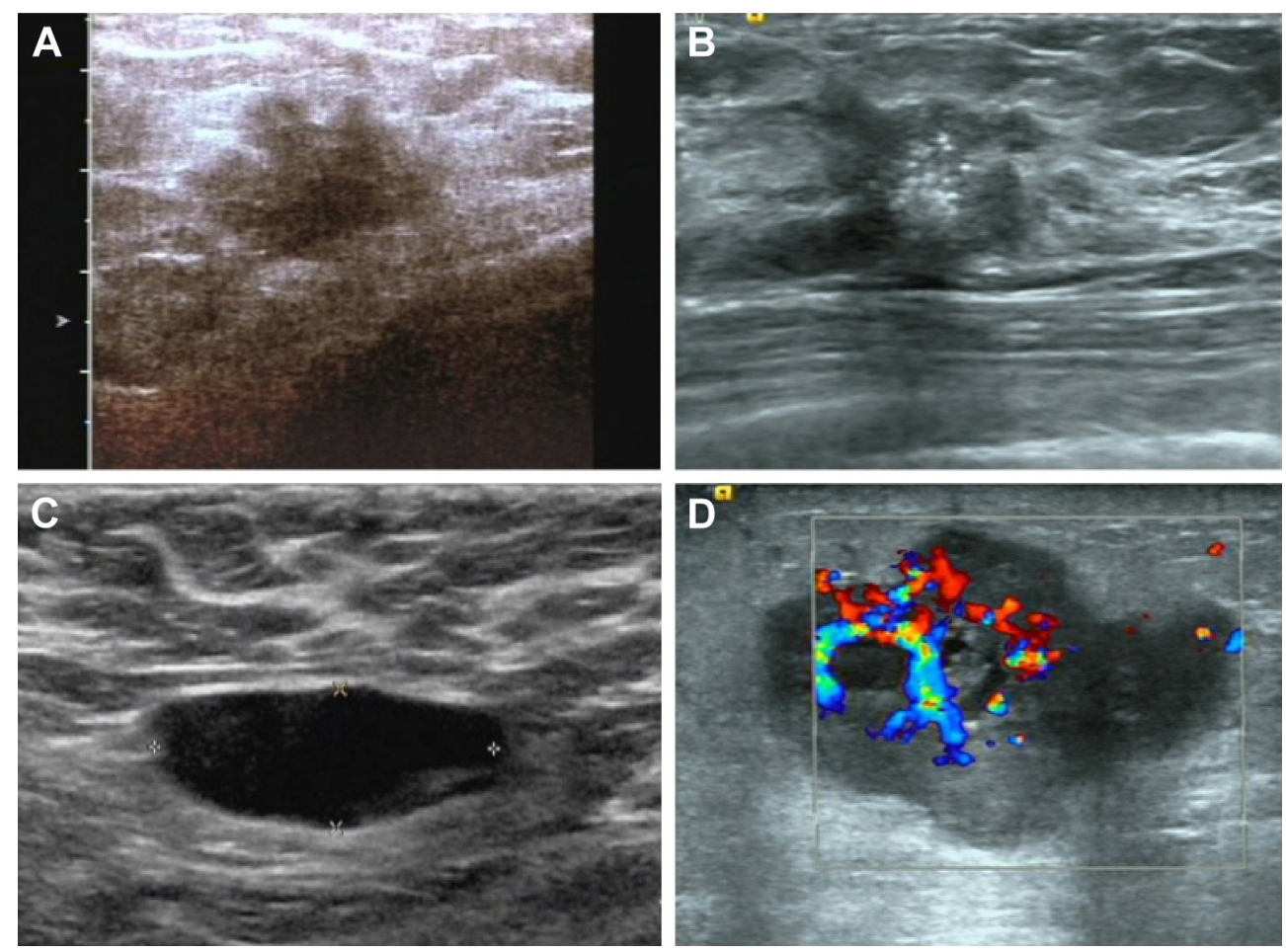

Figure I Ultrasonographic features of the patients with breast cancer.

Notes: (A) Edge burr sign. (B) Internal calcification. (C) Lymph node metastasis. (D) Blood flow grade III.

patients (Figure 1B). Lymph node metastasis was found in $105(56.5 \%)$ patients (Figure 1C). A total of 47 (25.3\%) patients had attenuated back echo. Blood flow signal of grade III was observed in 99 (53.2\%) patients (Figure 1D) and grades I-II in 87 (46.8\%) patients.

\section{The expression of SASHI in breast cancer}

In 186 patients with breast cancer, the positive expression of SASH1 was observed in $63(33.9 \%)$ patients (Figure 2A), and the other 123 patients $(66.1 \%)$ were diagnosed as negative expression (Figure 2B).

\section{The expression rate of SASHI in benign and malignant breast diseases}

There were no statistical differences in the age and gender between the patients with breast cancer and the controls $(P>0.05)$. Among 50 patients with ductal epithelial hyperplasia disease, positive expression of SASH1 was found in 31 patients $(62.0 \%)$. The positive expression rate of SASH1 was significantly decreased in patients with breast cancer (63/186, 33.9\%) compared with controls $(P<0.001)$. In addition, there was no statistical difference in positive expression rate of SASH1 between the different pathological types of breast cancer $(P=0.298$; Table 1$)$.
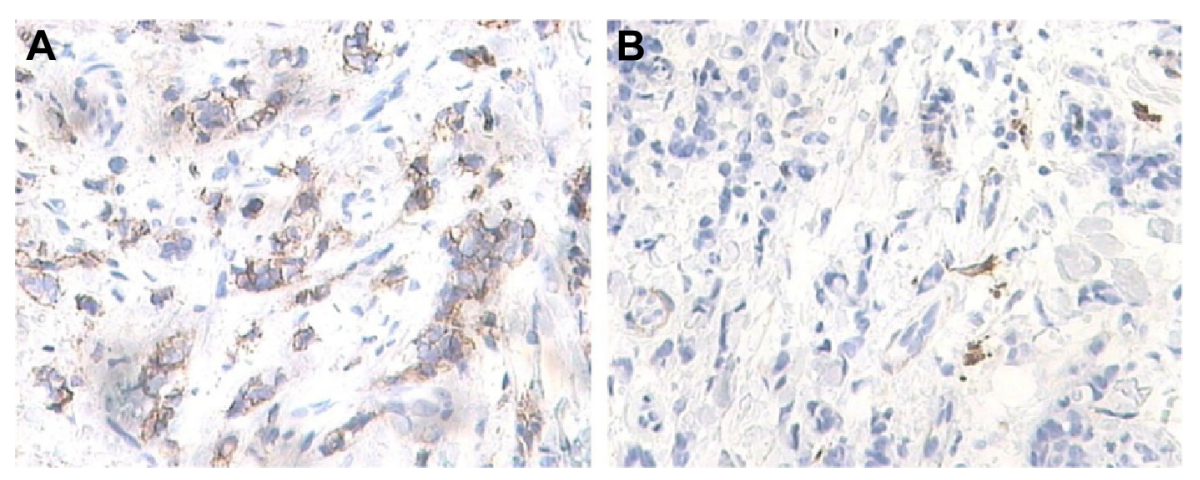

Figure 2 The expression of SASHI in breast cancer.

Notes: (A) The positive expression of SASHI was mainly observed in cytoplasm; (B) there was very little expression of SASHI in negative samples. Original magnification $\times 400$. 
Table I Expression of SASHI in benign and malignant breast diseases

\begin{tabular}{|c|c|c|c|c|c|c|}
\hline Patients & Subjects (n) & SASHI $^{+}$ & SASHI $^{-}$ & SASHI $^{+}$rate (\%) & $x^{2}$ & $P$-value \\
\hline Control & 50 & 31 & 19 & 62.0 & 13.010 & $<0.001$ \\
\hline Breast cancer & 186 & 63 & 123 & 33.9 & 2.421 & 0.298 \\
\hline Infiltrating ductal carcinoma & 153 & 48 & 105 & 31.4 & & \\
\hline Invasive lobular carcinoma & 18 & 8 & 10 & 44.4 & & \\
\hline $\begin{array}{l}\text { Invasive cribriform carcinoma } \\
\text { and mucinous carcinoma }\end{array}$ & 15 & 7 & 8 & 46.7 & & \\
\hline
\end{tabular}

Note: +, SASHI positive expression; -, negative expression.

\section{Relationship between SASHI expression and ultrasonographic features in breast cancer}

The positive expression rate of SASH1 was significantly decreased in patients with edge burr sign compared with those without edge burr sign $(P=0.025)$. Patients with lymph node metastasis had significantly decreased expression rate of SASH1 than those without metastasis $(P=0.007)$. Decreased expression rate of SASH1 was also found in patients who had a blood flow grade of III compared with those with I-II $(P=0.013)$. The difference in positive expression rate of SASH1 protein between the tumors with different size, shape, whether internal calcification, and weakened back echo was not statistically significant $(P>0.05$; Table 2$)$.

\section{Expression of SASHI correlates with edge burr sign, lymph node metastasis, and blood flow grade in patients with breast cancer}

Spearman's rank correlation analysis indicated that the expression of SASH1 was negatively correlated with edge burr sign of the patients with breast cancer $(r=-0.164$; 95\% CI: $\left.-0.314,-0.003, R^{2}=0.0269, P=0.025\right)$. In addition, the expression of SASH1 was also negatively correlated with lymph node metastasis ( $r=-0.196$; 95\% CI: $-0.333,-0.042$, $\left.R^{2}=0.0348, P=0.007\right)$ and blood flow grade $(r=-0.217$; 95\% CI: $\left.-0.369,-0.078, R^{2}=0.0471, P=0.003\right)$. However, there was no significant correlation between SASH1 expression and other ultrasonographic features, including size, shape, internal calcification, and back echo in patients with breast cancer (Table 3).

\section{Discussion}

Analysis of the correlation between the ultrasonographic features of breast cancer and the expression of SASH1 is beneficial to provide important reference for the postoperative treatment and the prognosis of the patients. In this study, we found that the positive expression rate of SASH1 was significantly decreased in patients with breast cancer (33.9\%) compared with patients having ductal epithelial hyperplasia disease. Zeller et $\mathrm{al}^{7}$ reported a low level expression of SASH1 in breast cancer. These data suggest that

Table 2 Relationship between SASHI expression and ultrasonographic features in breast cancer

\begin{tabular}{|c|c|c|c|c|c|c|}
\hline Ultrasonographic features & Subjects (n) & SASHI $^{+}$ & SASHI ${ }^{-}$ & $\mathrm{SASHI}^{+}$rate (\%) & $x^{2}$ & $P$-value \\
\hline Tumor size & & & & & 0.254 & 0.614 \\
\hline$\geq 2 \mathrm{~cm}$ & 75 & 27 & 48 & 36.0 & & \\
\hline$<2 \mathrm{~cm}$ & III & 36 & 75 & 32.4 & & \\
\hline Edge burr sign & & & & & 5.024 & 0.025 \\
\hline Yes & 118 & 33 & 85 & 28.0 & & \\
\hline No & 68 & 30 & 38 & 44.1 & & \\
\hline Shape & & & & & 1.310 & 0.252 \\
\hline Regular & 33 & 14 & 19 & 42.4 & & \\
\hline Irregular & 153 & 49 & 104 & 32.0 & & \\
\hline Internal calcification & & & & & 0.212 & 0.645 \\
\hline Yes & 90 & 29 & 61 & 32.2 & & \\
\hline No & 96 & 34 & 62 & 35.4 & & \\
\hline Lymph node metastasis & & & & & 7.162 & 0.007 \\
\hline Yes & 105 & 27 & 78 & 25.7 & & \\
\hline No & 81 & 36 & 45 & 44.4 & & \\
\hline Back echo & & & & & 0.107 & 0.743 \\
\hline Attenuation & 47 & 15 & 32 & 31.9 & & \\
\hline No attenuation & 139 & 48 & 91 & 34.5 & & \\
\hline Blood flow grade & & & & & 6.108 & 0.013 \\
\hline III & 99 & 24 & 75 & 24.2 & & \\
\hline I-II & 87 & 39 & 48 & 44.8 & & \\
\hline
\end{tabular}


Table 3 The expression of SASHI correlates with edge burr sign, lymph node metastasis, and blood flow grade

\begin{tabular}{lllll}
\hline $\begin{array}{l}\text { Ultrasonographic } \\
\text { features }\end{array}$ & $\boldsymbol{r}$ & $\mathbf{9 5 \%} \mathbf{C l}$ & $\boldsymbol{R}^{2}$ & $\boldsymbol{P}$-value \\
\hline Tumor size & 0.037 & $-0.119,0.176$ & 0.0014 & 0.616 \\
Edge burr sign & -0.164 & $-0.314,-0.003$ & 0.0269 & 0.025 \\
Shape & 0.084 & $-0.078,0.237$ & 0.0071 & 0.255 \\
Internal calcification & -0.034 & $-0.184,0.114$ & 0.0012 & 0.648 \\
Lymph node metastasis & -0.196 & $-0.333,-0.042$ & 0.0384 & 0.007 \\
Back echo & -0.024 & $-0.164,0.121$ & 0.0006 & 0.745 \\
Blood flow grade & -0.217 & $-0.369,-0.078$ & 0.0471 & 0.003 \\
\hline
\end{tabular}

SASH1 may play critical role in the development of breast cancer. Martini et $\mathrm{al}^{9}$ elucidated a potential anticancer mechanism of SASH1 including regulation of the adhesion and migration of cancer cells via interacting with the actin cytoskeleton. Reduced expression of SASH1 is correlated with increase in tumor size, aggressive growth, metastasis, and poor survival. ${ }^{7,10,11}$ In addition, the enhanced expression of SASH1 was correlated with reduced invasive ability in human U251 glioma cells. ${ }^{12}$ From the point of histologic type, there were no statistical differences in the positive rate of SASH1 expression when compared among groups of invasive ductal carcinoma, invasive lobular carcinoma, mucinous carcinoma, and invasive cribriform carcinoma. Burgess et $\mathrm{al}^{6}$ recently reported a significant difference in SASH1 expression between ER-positive and -negative breast cancers, but no statistical difference was found between human epidermal growth factor receptor 2 (HER2)-positive and -negative breast cancers.

The tumor showed a sign of burr, irregular boundary, internal calcification, and lymphatic metastasis, all of which indicated a malignant transformation. No correlation was found between SASH1 and tumor size, shape, or calcification in this study, suggesting that the expression of SASH1 is probably irrelevant to these macroscopically visible manifestations revealed by ultrasonic testing. Interestingly, we found that the positive rate of SASH1 expression was $28.0 \%$ in breast cancer patients with a sign of edge burr. However, the positive rate turned to be $44.1 \%$ in patients without a sign of edge burr. There was statistically significant difference between them. Spearman's rank correlation analysis showed that the edge burr sign had a significantly negative correlation with SASH1 expression. Furthermore, the positive rate of SASH1 expression was $25.7 \%$ in patients with lymphatic metastasis, while this positive rate was $44.4 \%$ in patients without lymphatic metastasis. The difference was also statistically significant. Relative analysis indicated a significantly negative correlation between lymphatic metastasis and SASH1 expression. The results mentioned above demonstrate that there is a certain correlation between expression of SASH1 and malignant characteristics of tumor, but the associated mechanisms still need further research.

In this study, the positive rate of SASH1 expression in stages I-II and III carcinoma based on the color Doppler blood flow classification was $44.8 \%$ and $24.2 \%$, respectively, which indicated a more abundant blood flow in breast cancer tissue and a lower positive rate of SASH1 expression. The intratumoral microvessel density has been regarded as one important indicator for quantitatively analyzing tumor angiogenesis, which can clearly reflect the intratumoral blood vessels state and tumor-induced angiogenesis ability. ${ }^{13}$ Some studies have shown that SASH1 is ubiquitously expressed in microvascular beds and acts as a scaffold for independently binding signaling molecules such as TRAF6, TAK1, and IKB kinase $\alpha$ and $\beta$. SASH1 functions to assemble a signaling complex to activate early endothelial responses upon TLR4 activation. ${ }^{14}$ The results in this study uncovered an inverse correlation between SASH1 expression in breast cancer tissue and microvascular flow, namely, a more abundant blood flow in breast cancer and a lower positive rate in SASH1 expression. This was possibly related to the different expression levels of SASH1 in tumor-derived neovascular endothelium and normal tissue vascular endothelium, suggesting that SASH1 may participate in the signaling transduction and regulation involved in tumor angiogenesis.

As for the posterior acoustic attenuation revealed in ultrasound testing, most scholars believe it is caused by enhanced absorption of sound energy owing to increase and disorganized arrangement of tumor interstitial collagen fiber. ${ }^{15}$ In this study, we did not observe a difference in positive rate of SASH1 expression existing between patients with appearance of posterior acoustic attenuation or without; moreover, no correlation was found between posterior acoustic attenuation and SASH1 expression. Thus, we consider a relationship existing between SASH1 and tumor microhemodynamics and there is no specific correlation between SASH1 and posterior acoustic attenuation.

The current data are beneficial to evaluate the clinicopathological characteristic of a patient with breast cancer. In addition, interpretation of the feature of SASH1 expression may help to use SASH1 as a treatment target for breast cancer in future. In this study, we investigated the relationship between SASH1 expression in breast cancer patients and ultrasonography features, but limits still exist in this study as follows: 1) No follow-up information is included. 2) A polyclonal antibody of SASH1 used in this study has potential possibility of nonspecific cross reactions. 3) The relationship between SASH1 expression and prognosis in breast cancer 
patients should be highlighted in future study, such as records and analysis of survival time and adverse events.

\section{Conclusion}

The expression of SASH1 was inversely correlated with some critical ultrasonographic features including edge burr sign, lymph node metastasis, and blood flow grade in patients with breast cancer. Our data suggest that decreased SASH1 expression appears to be associated with adverse clinical and imaging features in breast cancer.

\section{Acknowledgment}

This work was supported by the Foundation of Nantong Health Youth Fund Projects (WQ2014053).

\section{Disclosure}

The authors report no conflicts of interest in this work.

\section{References}

1. Siegel RL, Miller KD, Jemal A. Cancer statistics, 2015. CA Cancer J Clin. 2015;65(1):5-29.

2. Nash CE, Mavria G, Baxter EW, et al. Development and characterisation of a 3D multi-cellular in vitro model of normal human breast: a tool for cancer initiation studies. Oncotarget. 2015;6(15):13731-13741.

3. Stock AM, Klee F, Edlund K, et al. Gelsolin is associated with longer metastasis-free survival and reduced cell migration in estrogen receptorpositive breast cancer. Anticancer Res. 2015;35(10):5277-5285.

4. Liu R, Guo CX, Zhou HH. Network-based approach to identify prognostic biomarkers for estrogen receptor-positive breast cancer treatment with tamoxifen. Cancer Biol Ther. 2015;16(2):317-324.
5. Ren X, Liu Y, Tao Y, et al. Downregulation of SASH1 correlates with tumor progression and poor prognosis in ovarian carcinoma. Oncol Lett. 2016;11(5):3123-3130.

6. Burgess JT, Bolderson E, Saunus JM, et al. SASH1 mediates sensitivity of breast cancer cells to chloropyramine and is associated with prognosis in breast cancer. Oncotarget. 2016;14(10):12020.

7. Zeller C, Hinzmann B, Seitz S, et al. SASH1: a candidate tumor suppressor gene on chromosome 6q24.3 is downregulated in breast cancer. Oncogene. 2003;22(19):2972-2983.

8. Wilhelm A, McDonough MD, DePeri ER. Malignancy rates of nonmass-like enhancement on breast magnetic resonance imaging using American College of Radiology Breast Imaging Reporting and Data System descriptors. Breast J. 2012;18(6):523-526.

9. Martini M, Gnann A, Scheikl D, Holzmann B, Janssen K-P. The candidate tumor suppressor SASH1 interacts with the actin cytoskeleton and stimulates cell-matrix adhesion. Int J Biochem Cell Biol. 2011;43(11): $1630-1640$

10. Sheyu L, Hui L, Junyu Z, et al. Promoter methylation assay of SASH1 gene in breast cancer. J BUON. 2013;18(4):891-898.

11. Rimkus C, Martini M, Friederichs J, et al. Prognostic significance of downregulated expression of the candidate tumour suppressor gene SASH1 in colon cancer. Br J Cancer. 2006;95(10):1419-1423.

12. Yang L, Liu M, Gu Z, Chen J, Yan Y, Li J. Overexpression of SASH1 related to the decreased invasion ability of human glioma U251 cells. Tumor Biol. 2012;33(6):2255-2263.

13. Shang F, Liu M, Li B, et al. The anti-angiogenic effect of dexamethasone in a murine hepatocellular carcinoma model by augmentation of gluconeogenesis pathway in malignant cells. Cancer Chemother Pharmacol. 2016;77(5):1087-1096.

14. Dauphinee SM, Clayton A, Hussainkhel A, et al. SASH1 is a scaffold molecule in endothelial TLR4 signaling. J Immunol. 2013;191(2): 892-901.

15. Tamaki K, Ishida T, Miyashita M, et al. Breast ultrasonographic and histopathological characteristics without any mammographic abnormalities. Jpn J Clin Oncol. 2012;42(3):168-174.
OncoTargets and Therapy

\section{Publish your work in this journal}

OncoTargets and Therapy is an international, peer-reviewed, open access journal focusing on the pathological basis of all cancers, potential targets for therapy and treatment protocols employed to improve the management of cancer patients. The journal also focuses on the impact of management programs and new therapeutic agents and protocols on

\section{Dovepress}

patient perspectives such as quality of life, adherence and satisfaction The manuscript management system is completely online and includes a very quick and fair peer-review system, which is all easy to use. Visit http://www.dovepress.com/testimonials.php to read real quotes from published authors. 